\title{
The effect of hidden female smoking on the relationship between smoking and cardiovascular disease
}

\author{
Sang Won Hwang ${ }^{1 *}$, Hae Jeong Lee ${ }^{2 *}$, Cheol Hong Kim², \\ Sung Hoon Kim², Yechan Kyung ${ }^{2}$, Sang Taek Lee ${ }^{2}$, Ju Suk Lee ${ }^{2}$ \\ ${ }^{1}$ Department of Thoracic and Cardiovascular Surgery, Samsung Changwon Hospital, \\ Sungkyunkwan University School of Medicine, Changwon, Korea \\ ${ }^{2}$ Department of Pediatrics, Samsung Changwon Hospital, \\ Sungkyunkwan University School of Medicine, Changwon, Korea
}

\begin{abstract}
Background: Smoking is a known risk factor for cardiovascular morbidity and mortality, but several Korean studies have shown differing results on the association of current smoking status and the risk of cardiovascular disease (CVD). The aim of the present study was to investigate the association between smoking status and CVD (myocardial infarction and stroke) using national representative populationbased samples. The aim was also to investigate the effects of hidden smokers on the association between CVD and smoking.

Methods: Data were acquired from 28,620 participants (12,875 men and 15,745 women), age 19 years or older, who participated in the Korea National Health and Nutrition Examination Survey (KNHANES) conducted from 2008 to 2016.

Results: The multivariable logistic regression analysis showed that ex-smoking status was correlated with CVD when self-reported (odds ratio [OR]: 1.62; 95\% confidence interval [CI]: 1.20-2.19) and for survey-cotinine verified-smoking status (OR: 1.57; 95\% CI: 1.20-2.19). Interestingly, the present study showed current smoking was not significantly associated with CVD. For the effect of sex on smoking and $C V D$, self-reported and survey-cotinine-verified ex-smoking status were correlated with CVD in males (OR: 1.45; 95\% CI: 1.04-2.04 and OR: 1.43; 95\% CI: 1.02-2.02) and in females (OR: 2.74; 95\% CI: 1.59-4.71 and OR: 2.92; 95\% CI: 1.64-5.18). The ratios of cotinine-verified to self-reported smoking rates were 1.95 for women and 1.08 for men.

Conclusions: In the current study, while ex-smoking status was significantly associated with CVD, current smoking status was not. Female ex-smoking status had a higher adjusted odds ratio for CVD than males compared to non-smoking status. An effect of hidden female smoking was also found on the association between smoking status and CVD in Korean adults. (Cardiol J 2021; 28, 5: 716-727)

Key words: smoking, myocardial infarction, stroke, hidden smoker
\end{abstract}

\section{Introduction}

Smoking is a known risk factor of cardiovascular morbidity and mortality [1]. However, while more than 4000 chemical substances contained in a cigarette are known to have adverse effects on various cardiovascular diseases (CVD) [2], the pathophysiological mechanisms underlying the

Address for correspondence: Ju Suk Lee, MD, PhD, Department of Pediatrics, Samsung Changwon Hospital, Sungkyunkwan University School of Medicine, 158, Paryong-ro, Masanhoewon-gu, Changwon, Gyengsangnamdo, 51353, Korea, tel: 82-55-233-5932, fax: 82-55-233-5329, e-mail: ljs8952194@nate.com

Received: 18.06.2019 Accepted: 25.12.2019 Early publication date: 9.01.2020

*Sang Won Hwang and Hae Jeong Lee are co-first authors.

This article is available in open access under Creative Common Attribution-Non-Commercial-No Derivatives 4.0 International (CC BY-NC-ND 4.0) license, allowing to download articles and share them with others as long as they credit the authors and the publisher, but without permission to change them in any way or use them commercially. 
association between smoking and CVD have not been fully elucidated.

The CHANCES consortium (Consortium on Health and Ageing: Network of Cohorts in Europe and the United States) study showed a strong relationship between tobacco smoking and CVD [3]. This study used data from 10 cohort studies that showed a cardiovascular mortality hazard ratio of 2.07 (95\% confidence interval [CI]: 1.82-2.36) for current smokers and 1.37 (95\% CI: 1.25-1.49) for former smokers compared to participants who had never smoked. Another meta-analysis study based on prospective studies showed that the relative risk (RR) of sudden cardiac death due to CVD, including coronary heart disease and stroke, was 3.06 (95\% CI: 2.46-3.82) for current smokers and 1.38 (95\% CI: $1.20-1.60)$ for former smokers compared to participants who had never smoked [4].

Moreover, the INTERHEART study showed that current smoking status (odds ratio [OR] 2.87) was significantly associated with myocardial infarction (MI) [5], and a UK biobank study showed that the hazard ratios for MI were 3.46 (95\% CI: $3.02-3.98)$ in female current smokers and 2.23 (2.03-2.44) in male current smokers [6]. One metaanalysis study showed that the pooled relative risk of stroke associated with current smoking status vs. non-smoking status was 1.67 (95\% CI: 1.49-1.88) in men and 1.83 (95\% CI: 1.58-2.12) in women [7].

However, several Korean studies showed contrasting results on the association between current smoking status and CVD risk in adults $[8,9]$. A national study showed that smoking status was significantly associated with stroke (RR: $1.060,95 \%$ CI: $1.022-1.100$ ) but not MI (RR: $1.004,95 \%$ CI: 0.958-1.051) [8]. In addition, another national study showed that, compared to a non-smoking group of Korean adult males, the ORs (95\% CI) for physician-diagnosed stroke and $\mathrm{MI}$ in a current smoker group were $0.84(0.74-0.94)$ and $0.96(0.82-1.12)$, respectively, and 1.38 (1.24-1.53) and 1.45 (1.26-1.67), respectively, in a past smoker group [9].

Recently, a report showing that ratios of cotinine-verified to self-reported smoking rates were 2.36 for women and 1.12 for men in Korea [10]. In a previous report, an effect was found of hidden female smoking on the association between smoking and hypertension in Korean adults [11]. It is currently proposed that contradictory results from other countries may be due to hidden female smokers.

Therefore, the first aim of the study was to investigate the association between smoking status and CVD using different nationally representative population-based samples. The second aim was to investigate the effects of hidden female smokers on the association between CVD and smoking in Korean adults.

\section{Methods}

\section{Study population}

This study was based on data obtained from the 2008-2016 KNHANES study. KNHANES, which was a cross-sectional survey designed to examine the health and nutritional status of the non-institutionalized Korean population.

Of the total 76,909 KNHANES participants, 48,289 participants were excluded due to the following criteria: under 18 years old, no smoking history, no MI information, no stroke information, no urine cotinine test, no history of renal failure information, or serum creatinine $\geq 1.5 \mathrm{mg} / \mathrm{dL}$. The remaining 28,620 participants $(12,875$ men and 15,745 women) were included in the final analysis.

\section{General characteristics, anthropometry, and laboratory tests}

The contents of the questionnaire used in KNHANES included sex, age, marital status, employment status, education level, monthly family income, number of household members, residence area, and body mass index (BMI). The questionnaire also ascertained the presence of or history of hypertension, diabetes, MI, and stroke. The respondents' residential areas were categorized as urban (an administrative division of a city) or rural (not classified as an administrative division of a city). The definition of a city in Korea is a place where more than 50,000 people live. Monthly family income indicates monthly-equalized family income and was calculated by dividing the total family income by the square root of the number of household members. In KNHANES, monthly family income was classified into quartiles in order to determine the monthly household income level (1: low, 2: middle low, 3: middle high, and 4: high). Education level was defined as less than middle school, middle school, high school, and college or more. BMI was calculated as weight (kilograms) divided by height (meters squared) and was categorized into three groups: normal weight $\left(<23 \mathrm{~kg} / \mathrm{m}^{2}\right)$, overweight $\left(23-25 \mathrm{~kg} / \mathrm{m}^{2}\right)$, and obese $\left(\geq 25 \mathrm{~kg} / \mathrm{m}^{2}\right)$ [12].

Cigarette smoking status was divided into three categories: smoker, ex-smoker, and never smoked. Respondents who reported having consumed $\geq 100$ cigarettes in their lifetime or re- 
Table 1. Definition of survey-cotinine-verified smoking status.

\begin{tabular}{lcc}
\hline Self-reported smoking status & \multicolumn{2}{c}{ Cotinine-verified smoking status } \\
\cline { 2 - 3 } & Non-smoker $(\leq \mathbf{5 0} \mathbf{n g} / \mathbf{m L})$ & Smoker $(>\mathbf{5 0} \mathbf{n g} / \mathbf{m L})$ \\
\hline Non-smoker & Non-smoker $(\mathrm{n}=16,595)$ & Smoker $(\mathrm{n}=\mathbf{8 1 3})$ \\
Ex-smoker & Ex-smoker $(\mathrm{n}=\mathbf{4 7 1 2})$ & Smoker $(\mathrm{n}=\mathbf{5 1 7})$ \\
Smoker & Smoker $(\mathrm{n}=176)$ & Smoker $(\mathrm{n}=5,807)$ \\
\hline
\end{tabular}

sponded "yes" to the question, "Do you smoke cigarettes now?" were regarded as smokers. Participants answering "no" to the same question were classified as ex-smokers. Respondents who consumed $<100$ cigarettes in their lifetime were regarded as never having smoked.

Urinary cotinine was measured by tandem mass spectrometry with a tandem mass API 4000 (Applied Biosystems, Carlsbad, CA, USA) and by gas chromatography and mass spectrometry with a Perkin Elmer Clarus 600T (PerkinElmer, Turku, Finland). Respondents with urinary cotinine concentrations $\geq 50 \mathrm{ng} / \mathrm{mL}$ were considered cotinine-verified smokers, and those with cotinine levels $<50 \mathrm{ng} / \mathrm{mL}$ were cotinine-verified non-smokers [13].

To define MI and stroke, the following survey question was used in this study: "Have you ever been diagnosed with myocardial infarction or stroke by a physician?" For convenience, CVD to MI and stroke were limited. In a similar questionnaire, blood pressure (BP) measurements and medication history were used to define hypertension with the following question: "Have you ever been diagnosed with hypertension by a physician or take a medicine now?" Seated BP was measured using a standardized automated oscillometric device after a 5 -min rest period. If BP was abnormally high or low, BP was reassessed with a mercury sphygmomanometer by a trained nurse. Hypertension was defined as having elevated BP (systolic $\mathrm{BP}$ [SBP] $\geq 140 \mathrm{mmHg}$ and/or diastolic BP [DBP] $\geq 90 \mathrm{mmHg}$ ). Participants currently prescribed anti-hypertensive medication were also considered to be hypertensive. Diabetes was defined as a fasting blood glucose (FBG) of $126 \mathrm{mg} / \mathrm{dL}$. Participants who reported being diagnosed with diabetes by a physician and prescribed diabetes medication including insulin were also considered to be diabetic.

To better understand the link between MI, stroke, and smoking, a new variable was created for defining smoking status (survey-cotinine-verified smoking status [SCS]) (Table 1). It was assumed that smoking in this variable included all smoking types, including light, intermittent, passive, hidden, and active heavy smoking (Table 1).

\section{Statistical analysis}

All statistical analyses was conducted using the Statistical Package for the Social Sciences (SPSS) complex sample procedures since KNHANES data were collected through a representative, stratified, and clustered sampling method. Values are presented as number of participants or estimates (95\% CI). Data are expressed as the mean \pm standard deviation (SD) and as the median for continuous variables and as percentages $(\%)$ for categorical variables.

Calculations of crude odd ratios and $95 \% \mathrm{CI}$ values for CVD in relation to potential risk factors were performed using univariable logistic regression models. Finally, multivariable logistic regression analysis was carried out to identify the relationships between risk factors and CVD to evaluate the relationship of self-reported and cotinine-verified smoking status with CVD. Sex, age, marital status, employment status, education level, monthly family income, number of household members, residence area, smoking status, BMI, presence of hypertension and presence of diabetes were corrected for in the final multivariable logistic regression model.

Statistical analysis was conducted using SPSS version 21.0 (SPSS Inc. Chicago, IL, US). For all analyses, $\mathrm{p}$ values were two-tailed, and a $\mathrm{p}$ value $<0.05$ was considered statistically significant.

\section{Results}

\section{Clinical characteristics}

A total of 28,620 subjects from the KNHANES were included in this study. Mean age was $49.55 \pm$ \pm 16.35 years. Slightly over half $(52.3 \%)$ were male. The majority of participants were married (75.0\%), employed (64.2\%), and had an education level of high school or higher (73.9\%). Most subjects lived in urban areas (83.1\%) (Table 2). 
Table 2. Demographic characteristics of subjects.

\begin{tabular}{|c|c|c|}
\hline Characteristics & Sample size & Estimate $\%(95 \% \mathrm{Cl})$ \\
\hline \multicolumn{3}{|l|}{$\operatorname{Sex}(n=28,620)$} \\
\hline Male & 12,875 & $52.29(51.74-52.85)$ \\
\hline Female & 15,745 & $47.71(47.15-48.26)$ \\
\hline \multicolumn{3}{|l|}{ Age $(n=28,620)$} \\
\hline$<50$ & 14,332 & $62.00(61.04-62.97)$ \\
\hline $50-59$ & 5515 & $18.89(18.27-19.51)$ \\
\hline $60-69$ & 5000 & $11.30(10.85-11.77)$ \\
\hline$\geq 70$ & 3773 & $7.81(7.41-8.24)$ \\
\hline \multicolumn{3}{|l|}{ Marital status $(n=26,740)$} \\
\hline Married & 20,644 & $74.95(74.03-75.84)$ \\
\hline Single (separated or divorced) & 3491 & $10.10(9.61-10.62)$ \\
\hline Never-married & 2605 & $14.95(14.15-15.80)$ \\
\hline \multicolumn{3}{|l|}{ Employment status ( $n=28,541$ ) } \\
\hline Employed & 17,307 & $64.19(63.40-64.97)$ \\
\hline Unemployed & 11,234 & $35.81(35.03-36.60)$ \\
\hline \multicolumn{3}{|l|}{ Education level ( $n=28,541$ ) } \\
\hline$<$ High school & 9918 & $26.10(25.18-27.05)$ \\
\hline High school & 9755 & $38.94(38.08-39.80)$ \\
\hline$>$ High school & 8868 & $34.96(33.89-36.04)$ \\
\hline \multicolumn{3}{|l|}{ Monthly family income ( $n=28,310$ ) } \\
\hline$<25^{\text {th }}$ & 5284 & $14.80(14.06-15.57)$ \\
\hline $25-50^{\text {th }}$ & 7149 & $24.85(23.96-25.76)$ \\
\hline $50-75^{\text {th }}$ & 7851 & $29.64(28.68-30.61)$ \\
\hline$\geq 75^{\text {th }}$ & 8026 & $30.72(29.40-32.06)$ \\
\hline \multicolumn{3}{|l|}{ Number of household members ( $n=28,608$ ) } \\
\hline 1 & 2521 & $7.26(6.68-7.88)$ \\
\hline 2 & 7695 & $21.58(20.84-22.33)$ \\
\hline 3 & 6968 & $26.67(25.82-27.54)$ \\
\hline 4 & 7725 & $30.92(29.95-31.91)$ \\
\hline$\geq 5$ & 3699 & $13.58(12.80-14.39)$ \\
\hline \multicolumn{3}{|l|}{ Residence area ( $n=28,620$ ) } \\
\hline Urban & 22,156 & $83.14(81.04-85.05)$ \\
\hline Rural & 6104 & $16.86(14.95-18.96)$ \\
\hline \multicolumn{3}{|l|}{ Smoking status } \\
\hline \multicolumn{3}{|l|}{ Self-reported 1 ( $n=28,620)$} \\
\hline Non-smoker & 17,408 & $56.41(55.73-57.09)$ \\
\hline Ex-smoker & 5229 & $18.05(17.55-18.56)$ \\
\hline Smoker & 5983 & $25.54(24.87-26.22)$ \\
\hline \multicolumn{3}{|l|}{ Cotinine-verified $(n=28,620$ ) } \\
\hline Non-smoker & 21,483 & $70.33(69.58-71.07)$ \\
\hline Smoker & 7137 & $29.67(28.93-30.42)$ \\
\hline \multicolumn{3}{|l|}{ Survey-cotinine verified $(n=28,620)$} \\
\hline Non-smoker & 16,595 & $53.57(52.85-54.29)$ \\
\hline Ex-smoker & 4712 & $15.98(15.49-16.47)$ \\
\hline Smoker & 7313 & $30.45(29.72-31.20)$ \\
\hline \multicolumn{3}{|l|}{ Body mass index $(n=28,620)$} \\
\hline$<23$ & 12,318 & $43.64(42.90-44.37)$ \\
\hline $23-25$ & 6812 & $23.71(23.13-24.30)$ \\
\hline$\geq 25$ & 9415 & 32.66 (31.95-33.37) \\
\hline Hypertension $(n=28,620)$ & 7917 & $23.69(23.04-24.36)$ \\
\hline Diabetes $(n=28,620)$ & 3082 & $8.80(8.42-9.20)$ \\
\hline Myocardial infarct or stroke $(n=28,620)$ & 811 & $1.98(1.81-2.16)$ \\
\hline Myocardial infarct & 236 & $0.60(0.51-0.70)$ \\
\hline Stroke & 599 & $1.44(1.30-1.60)$ \\
\hline
\end{tabular}


Table 3. Self-reported and cotinine-verified smoking status in male and female participants.

\begin{tabular}{lccc}
\hline $\begin{array}{l}\text { Self-reported } \\
\text { smoking status }\end{array}$ & Total & Cotinine-verified smoking status (estimate \% [95\% CI]) \\
\cline { 3 - 4 } & & Non-smoker & Smoker \\
\hline Male (n= 12,875) & & $53.4(52.3-54.4)$ & $46.6(45.6-47.7)$ \\
Total & & $24.8(23.9-25.7)$ & $1.2(1.0-1.4)$ \\
Non-smoker & $26.0(25.0-26.9)$ & $27.4(26.5-28.3)$ & $3.2(2.9-3.6)$ \\
Ex-smoker & $30.6(29.6-31.5)$ & $1.2(1.0-1.5)$ & $42.3(41.2-43.3)$ \\
Smoker & $43.5(42.4-44.5)$ & & $11.1(10.4-11.8)$ \\
Female (n= 15,745) & & $88.9(88.2-89.6)$ & $4.7(4.2-5.2)$ \\
Total & & $85.1(84.3-85.9)$ & $0.8(0.7-1.0)$ \\
Non-smoker & $89.8(89.1-90.4)$ & $3.5(3.2-3.9)$ & $5.6(5.1-6.1)$ \\
Ex-smoker & $4.3(4.0-4.8)$ & $0.3(0.2-0.4)$ & \\
Smoker & $5.9(5.4-6.4)$ & & \\
\hline
\end{tabular}

\section{Smoking status}

Self-reported smoking prevalence was $25.5 \%$, whereas the prevalence of cotinine-verified smokers in the overall population was $29.7 \%$ (Table 2). Cotinine-verified smoking prevalence for men and women was $43.3 \%(5,578 / 12,875)$ and $9.9 \%$ $(1,559 / 15,745)$, respectively (Table 3$)$. The overall prevalence of self-reported ex-smokers and current smokers was $18.1 \%$ and $25.5 \%$, the percentages were $30.6 \%$ and $43.5 \%$ in men and $4.3 \%$ and $5.9 \%$ in women (Tables 2,3 ). Of the 7,137 cotinine-verified male and female smokers, 813 $(11.4 \%)$ were self-reported non-smokers and 517 $(7.2 \%)$ were self-reported ex-smokers. Specifically, $2.3 \%$ and $7.3 \%$ of male cotinine-verified smokers were self-reported non-smokers and ex-smokers, respectively, whereas $43.7 \%$ and $7.1 \%$ of female cotinine-verified smokers were self-reported non-smokers and ex-smokers, respectively (Table 3). The ratios of cotinine-verified to self-reported smoking rates were $1.95(1,559 / 801)$ for women and $1.08(5,578 / 5,182)$ for men (Table 3$)$.

\section{Relationship between MI \\ or stroke and smoking}

In a univariate analysis, subjects with MI or stroke were prone to be male $(\mathrm{p}<0.01)$, older $(p<0.01)$, single (separated or divorced) $(p<0.01)$, unemployed $(\mathrm{p}<0.01)$, less educated $(\mathrm{p}<0.01)$, obese $(p<0.01)$, have a lower monthly family income $(\mathrm{p}<0.01)$, have fewer household members $(\mathrm{p}<0.01)$, living in a rural setting $(\mathrm{p}<0.01)$, have hypertension $(\mathrm{p}<0.01)$, and have diabetes $(\mathrm{p}<0.01)$ compared to subjects without CVD (Table 4). For self-reported smoking status, ex-smoking status was significantly correlated with CVD $(\mathrm{p}<0.01)$, and for SCS, ex-smoking status was also correlated with CVD ( $\mathrm{p}<0.01)$. However, for all three types of smoking status, current smoking status was not significantly correlated with CVD using univariate statistical analysis $(\mathrm{p}>0.05)$ (Table 4$)$.

The multivariate logistic regression analysis showed that for self-reported smoking status, ex-smoking status was correlated with CVD (OR: 1.62; 95\% CI: 1.20-2.19). Also, for SCS, ex-smoking status was correlated with CVD (OR: 1.57; 95\% CI: 1.20-2.19) (Table 5).

To determine the effects of sex on smoking and CVD, sex differences were analyzed separately. For male participants, self-reported status and survey-cotinine-verified ex-smoking status were correlated with CVD (OR: $1.45 ; 95 \% \mathrm{CI}$ : 1.04-2.04, OR: 1.43; 95\% CI: 1.02-2.02). Female self-reported status and survey-cotinine-verified ex-smoking status were also correlated with CVD (OR: 2.74; 95\% CI: 1.59-4.71, OR: 2.92; 95\% CI: 1.64-5.18) (Tables 6, 7). Therefore, the current results indicate that sex affected the incidence of CVD in this study.

\section{Discussion}

The main findings of this study showed that, while ex-smoking status was significantly associated with CVDs, current smoking status was not, and female ex-smoking status had higher adjusted OR for CVD than males compared to non-smoking status. In addition, there was an effect of hidden female smoking on the association between smoking status and CVD in Korean adults.

Smoking is an established risk factor for cardiovascular morbidity and mortality [1]. 
Table 4. Crude odds ratios and $95 \%$ confidence intervals $(\mathrm{Cl})$ for myocardial infarction and stroke prevalence.

\begin{tabular}{|c|c|c|c|}
\hline \multirow[t]{2}{*}{ Characteristics } & \multicolumn{3}{|c|}{ Odds ratio $(95 \% \mathrm{Cl})$} \\
\hline & Total & Male & Female \\
\hline \multicolumn{4}{|l|}{ Sex } \\
\hline Male & $1.40(1.19-1.65)$ & - & - \\
\hline Female & Reference & - & - \\
\hline \multicolumn{4}{|l|}{ Age } \\
\hline$<50$ & Reference & Reference & Reference \\
\hline $50-59$ & $8.10(5.48-11.96)$ & $7.65(4.78-12.24)$ & $11.07(5.45-22.47)$ \\
\hline $60-69$ & $21.90(15.38-31.19)$ & $21.03(13.81-32.02)$ & $31.48(16.18-61.26)$ \\
\hline$\geq 70$ & $33.92(24.05-47.85)$ & $28.01(18.40-42.64)$ & $63.78(33.44-121.65)$ \\
\hline \multicolumn{4}{|l|}{ Marital status } \\
\hline Married & Reference & Reference & Reference \\
\hline Single (separated or divorced) & $2.28(1.87-2.80)$ & $1.94(1.30-2.87)$ & $3.48(2.70-4.49)$ \\
\hline Never-married & $0.15(0.08-0.29)$ & $0.15(0.07-0.31)$ & $0.10(0.02-0.40)$ \\
\hline \multicolumn{4}{|l|}{ Employment status } \\
\hline Employed & Reference & Reference & Reference \\
\hline Unemployed & $2.98(2.50-3.55)$ & 3.74 (2.99-4.68) & $3.44(2.61-4.55)$ \\
\hline \multicolumn{4}{|l|}{ Education } \\
\hline$<$ High school & $8.62(6.45-11.50)$ & 7.44 (5.33-10.39) & $20.45(10.76-38.86)$ \\
\hline High school & $1.90(1.37-2.63)$ & $1.83(1.27-2.64)$ & $2.62(1.31-5.24)$ \\
\hline$>$ High school & Reference & Reference & Reference \\
\hline \multicolumn{4}{|l|}{ Monthly family income } \\
\hline$<25^{\text {th }}$ & $6.30(4.78-8.30)$ & $5.70(4.04-8.04)$ & $9.06(5.89-13.92)$ \\
\hline $25-50^{\text {th }}$ & $2.13(1.58-2.87)$ & $1.80(1.24-2.61)$ & $3.17(2.01-5.00)$ \\
\hline $50-75^{\text {th }}$ & $1.44(1.06-1.96)$ & $1.24(0.85-1.82)$ & $2.01(1.23-3.26)$ \\
\hline$\geq 75^{\text {th }}$ & Reference & Reference & Reference \\
\hline \multicolumn{4}{|l|}{ Number of household members } \\
\hline 1 & $2.25(1.60-3.18)$ & $1.29(0.74-2.18)$ & $3.91(2.36-6.47)$ \\
\hline 2 & $2.40(1.79-3.22)$ & $2.34(1.62-3.40)$ & $2.50(1.56-3.99)$ \\
\hline 3 & $0.93(0.67-1.30)$ & $0.91(0.60-1.38)$ & $0.92(0.55-1.54)$ \\
\hline 4 & $0.56(0.39-0.80)$ & $0.56(0.36-0.89)$ & $0.50(0.27-0.91)$ \\
\hline$\geq 5$ & Reference & Reference & Reference \\
\hline \multicolumn{4}{|l|}{ Residence area } \\
\hline Urban & Reference & Reference & Reference \\
\hline Rural & $1.53(1.25-1.87)$ & $1.39(1.07-1.81)$ & $1.74(1.33-2.29)$ \\
\hline \multicolumn{4}{|l|}{ Smoking status } \\
\hline \multicolumn{4}{|l|}{ Self-reported } \\
\hline Non-smoker & Reference & Reference & Reference \\
\hline Ex-smoker & $2.69(2.24-3.24)$ & $3.30(2.43-4.49)$ & $2.00(1.26-3.16)$ \\
\hline Smoker & $0.99(0.79-1.24)$ & $1.23(0.87-1.72)$ & $0.61(0.35-1.08)$ \\
\hline \multicolumn{4}{|l|}{ Cotinine-verified } \\
\hline Non-smoker & Reference & Reference & Reference \\
\hline Smoker & $0.67(0.55-0.83)$ & $0.56(0.43-0.71)$ & $0.50(0.32-0.79)$ \\
\hline \multicolumn{4}{|l|}{ Survey-cotinine verified } \\
\hline Non-smoker & Reference & Reference & Reference \\
\hline Ex-smoker & $2.79(2.30-3.38)$ & $3.37(2.46-4.60)$ & $2.25(1.39-3.63)$ \\
\hline Smoker & $0.92(0.74-1.15)$ & $1.19(0.85-1.67)$ & $0.53(0.34-0.84)$ \\
\hline \multicolumn{4}{|l|}{ Body mass index } \\
\hline$<23$ & Reference & Reference & Reference \\
\hline $23-25$ & 1.49 (1.19-1.87) & $1.13(0.84-1.51)$ & $1.98(1.42-2.77)$ \\
\hline$\geq 25$ & $1.88(1.55-2.29)$ & 1.35 (1.03-1.77) & $2.70(2.02-3.59)$ \\
\hline \multicolumn{4}{|l|}{ Hypertension } \\
\hline No & Reference & Reference & Reference \\
\hline Yes & $5.60(4.71-6.67)$ & $4.24(3.34-5.38)$ & $8.18(6.24-10.74)$ \\
\hline \multicolumn{4}{|l|}{ Diabetes } \\
\hline No & Reference & Reference & Reference \\
\hline Yes & $5.36(4.47-6.42)$ & $5.89(4.62-7.50)$ & $4.41(3.36-5.78)$ \\
\hline
\end{tabular}


Table 5. Adjusted odds ratios and $95 \%$ confidence intervals for myocardial infarction and stroke prevalence.

\begin{tabular}{|c|c|c|c|}
\hline Characteristics & Self-reported & Cotinine-verified & Survey-cotinine verified \\
\hline \multicolumn{4}{|l|}{ Sex } \\
\hline Male & $1.70(1.26-2.27)$ & $2.39(1.93-2.94)$ & $1.78(1.35-2.35)$ \\
\hline Female & Reference & Reference & Reference \\
\hline \multicolumn{4}{|l|}{ Age } \\
\hline$<50$ & Reference & Reference & Reference \\
\hline $50-59$ & 4.88 (3.12-7.65) & $4.89(3.11-7.69$ & $4.84(3.09-7.59)$ \\
\hline $60-69$ & $8.52(5.41-13.44)$ & $8.52(5.38-13.48)$ & $8.42(5.33-13.30)$ \\
\hline$\geq 70$ & $9.81(6.05-15.92)$ & $9.78(6.01-15.92)$ & $9.64(5.93-15.67)$ \\
\hline \multicolumn{4}{|l|}{ Marital status } \\
\hline Married & Reference & Reference & Reference \\
\hline Single (separated or divorced) & $0.97(0.73-1.28)$ & $0.99(0.75-1.30)$ & $0.97(0.74-1.28)$ \\
\hline Never-married & $0.75(0.36-1.54)$ & $0.69(0.34-1.43)$ & $0.74(0.36-1.52)$ \\
\hline \multicolumn{4}{|l|}{ Employment status } \\
\hline Employed & Reference & Reference & Reference \\
\hline Unemployed & $1.90(1.55-2.33)$ & $1.93(0.57-2.38)$ & $1.90(1.55-2.34)$ \\
\hline \multicolumn{4}{|l|}{ Education } \\
\hline$<$ High school & $1.78(1.29-2.45)$ & $1.80(1.30-2.48)$ & $1.79(1.30-2.47)$ \\
\hline High school & $1.36(0.96-1.93)$ & $1.38(0.98-1.94)$ & $1.37(0.97-1.93)$ \\
\hline$>$ High school & Reference & Reference & Reference \\
\hline \multicolumn{4}{|l|}{ Monthly family income } \\
\hline$<25^{\text {th }}$ & $1.69(1.24-2.30)$ & $1.70(1.25-2.32)$ & $1.70(1.25-2.32)$ \\
\hline $25-50^{\text {th }}$ & $1.25(0.91-1.72)$ & $1.25(0.91-1.72)$ & $1.25(0.91-1.72)$ \\
\hline $50-75^{\text {th }}$ & $1.27(0.92-1.76)$ & $1.28(0.93-1.77)$ & $1.27(0.92-1.76)$ \\
\hline$\geq 75^{\text {th }}$ & Reference & Reference & Reference \\
\hline \multicolumn{4}{|l|}{ Number of household members } \\
\hline 1 & $0.94(0.65-1.37)$ & $0.95(0.65-1.38)$ & $0.94(0.65-1.38)$ \\
\hline 2 & $0.95(0.68-1.32)$ & $0.96(0.69-1.34)$ & $0.95(0.68-1.33)$ \\
\hline 3 & $0.82(0.58-1.17)$ & $0.82(0.58-1.18)$ & $0.82(0.58-1.18)$ \\
\hline 4 & $0.82(0.56-1.20)$ & $0.82(0.56-1.20)$ & $0.82(0.56-1.21)$ \\
\hline$\geq 5$ & Reference & Reference & Reference \\
\hline \multicolumn{4}{|l|}{ Residence area } \\
\hline Urban & Reference & Reference & Reference \\
\hline Rural & $1.13(0.91-1.40)$ & $1.12(0.90-1.39)$ & $1.13(0.91-1.40)$ \\
\hline \multicolumn{4}{|l|}{ Smoking status } \\
\hline \multicolumn{4}{|l|}{ Self-reported } \\
\hline Non-smoker & Reference & - & - \\
\hline Ex-smoker & $1.62(1.20-2.19)$ & - & - \\
\hline Smoker & $1.25(0.92-1.70)$ & - & - \\
\hline \multicolumn{4}{|l|}{ Cotinine-verified } \\
\hline Non-smoker & - & Reference & - \\
\hline Smoker & - & $0.88(0.69-1.11)$ & - \\
\hline \multicolumn{4}{|l|}{ Survey-cotinine verified } \\
\hline Non-smoker & - & - & Reference \\
\hline Ex-smoker & - & - & $1.57(1.16-2.12)$ \\
\hline Smoker & - & - & $1.15(0.87-1.53)$ \\
\hline \multicolumn{4}{|l|}{ Body mass index } \\
\hline$<23$ & Reference & Reference & Reference \\
\hline $23-25$ & $1.10(1.20-2.19)$ & $1.10(0.87-1.40)$ & $1.10(0.86-1.40)$ \\
\hline$\geq 25$ & $1.25(0.92-1.70)$ & $1.24(0.99-1.56)$ & $1.24(0.99-1.56)$ \\
\hline \multicolumn{4}{|l|}{ Hypertension } \\
\hline No & Reference & Reference & Reference \\
\hline Yes & $2.06(1.69-2.50)$ & $2.05(1.69-2.50)$ & $2.05(1.69-2.49)$ \\
\hline \multicolumn{4}{|l|}{ Diabetes } \\
\hline No & Reference & Reference & Reference \\
\hline Yes & $1.85(1.52-2.25)$ & $1.86(1.53-2.27)$ & $1.85(1.52-2.26)$ \\
\hline
\end{tabular}

Adjusted for sex, age, marital status, employment status, education level, monthly family income, number of household members, residence area, smoking status, body mass index, hypertension and diabetes. 
Table 6. Adjusted odds ratios and $95 \%$ confidence intervals for myocardial infarction and stroke prevalence in male participants.

\begin{tabular}{|c|c|c|c|}
\hline Characteristics & Self-reported & Cotinine-verified & Survey-cotinine verified \\
\hline \multicolumn{4}{|l|}{ Age } \\
\hline$<50$ & Reference & Reference & Reference \\
\hline $50-59$ & $4.63(2.66-8.06)$ & $4.70(2.70-8.20)$ & $4.60(2.64-8.01)$ \\
\hline $60-69$ & $8.65(4.93-15.18)$ & $8.80(5.01-15.45)$ & 8.57 (4.87-15.07) \\
\hline$\geq 70$ & $8.09(4.33-15.10)$ & 8.17 (4.37-15.27) & 7.97 (4.25-14.94) \\
\hline \multicolumn{4}{|l|}{ Marital status } \\
\hline Married & Reference & Reference & Reference \\
\hline Single (separated or divorced) & $1.01(0.62-1.65)$ & $1.02(0.63-1.67)$ & $1.01(0.62-1.65)$ \\
\hline Never-married & $0.72(0.30-1.74)$ & $0.67(0.28-1.61)$ & $0.72(0.30-1.72)$ \\
\hline \multicolumn{4}{|l|}{ Employment status } \\
\hline Employed & Reference & Reference & Reference \\
\hline Unemployed & $1.92(1.44-2.56)$ & $1.96(1.47-2.61)$ & $1.93(1.45-2.57)$ \\
\hline \multicolumn{4}{|l|}{ Education } \\
\hline$<$ High school & $1.74(1.20-2.52)$ & $1.76(1.22-2.55)$ & $1.75(1.21-2.53)$ \\
\hline High school & $1.39(0.93-2.07)$ & $1.40(0.94-2.09)$ & $1.39(0.93-2.08)$ \\
\hline$>$ High school & Reference & Reference & Reference \\
\hline \multicolumn{4}{|l|}{ Monthly family income } \\
\hline$<25^{\text {th }}$ & $1.71(1.14-2.57)$ & $1.71(1.14-2.57)$ & $1.72(1.15-2.58)$ \\
\hline $25-50^{\text {th }}$ & $1.11(0.75-1.65)$ & $1.11(0.74-1.65)$ & $1.11(0.75-1.66)$ \\
\hline $50-75^{\text {th }}$ & $1.15(0.77-1.72)$ & $1.15(0.77-1.73)$ & $1.15(0.76-1.72)$ \\
\hline$\geq 75^{\text {th }}$ & Reference & Reference & Reference \\
\hline \multicolumn{4}{|l|}{ Number of household members } \\
\hline 1 & $0.77(0.41-1.45)$ & $0.78(0.41-1.46)$ & $0.77(0.41-1.45)$ \\
\hline 2 & $0.90(0.59-1.38)$ & $0.92(0.60-1.40)$ & $0.91(0.59-1.39)$ \\
\hline 3 & $0.78(0.50-1.22)$ & $0.78(0.50-1.23)$ & $0.78(0.50-1.23)$ \\
\hline 4 & $0.84(0.52-1.36)$ & $0.85(0.52-1.37)$ & $0.85(0.52-1.37)$ \\
\hline$\geq 5$ & Reference & Reference & Reference \\
\hline \multicolumn{4}{|l|}{ Residence area } \\
\hline Urban & Reference & Reference & Reference \\
\hline Rural & $1.07(0.80-1.42)$ & $1.06(0.80-1.41)$ & $1.07(0.80-1.42)$ \\
\hline \multicolumn{4}{|l|}{ Smoking status } \\
\hline \multicolumn{4}{|l|}{ Self-reported } \\
\hline Non-smoker & Reference & - & - \\
\hline Ex-smoker & $1.45(1.04-2.04)$ & - & - \\
\hline Smoker & $1.17(0.81-1.67)$ & - & - \\
\hline \multicolumn{4}{|l|}{ Cotinine-verified } \\
\hline Non-smoker & - & Reference & - \\
\hline Smoker & - & $0.87(0.65-1.15)$ & - \\
\hline \multicolumn{4}{|l|}{ Survey-cotinine verified } \\
\hline Non-smoker & - & - & Reference \\
\hline Ex-smoker & - & - & $1.43(1.02-2.02)$ \\
\hline Smoker & - & - & $1.12(0.78-1.60)$ \\
\hline \multicolumn{4}{|l|}{ Body mass index } \\
\hline$<23$ & Reference & Reference & Reference \\
\hline $23-25$ & $1.11(0.80-1.53)$ & $1.11(0.80-1.54)$ & $1.10(0.80-1.53)$ \\
\hline$\geq 25$ & $1.37(0.99-1.89)$ & 1.37 (0.99-1.89) & $1.36(0.98-1.89)$ \\
\hline \multicolumn{4}{|l|}{ Hypertension } \\
\hline No & Reference & Reference & Reference \\
\hline Yes & $1.84(1.41-2.40)$ & $1.85(1.41-2.41)$ & $1.84(1.41-2.39)$ \\
\hline \multicolumn{4}{|l|}{ Diabetes } \\
\hline No & Reference & Reference & Reference \\
\hline Yes & $2.26(1.72-2.97)$ & $2.29(1.74-3.01)$ & $2.26(1.72-2.98)$ \\
\hline
\end{tabular}

Adjusted for age, marital status, employment status, education level, monthly family income, number of household members, residence area, smoking status, body mass index, hypertension and diabetes. 
Table 7. Adjusted odds ratios and $95 \%$ confidence intervals for myocardial infarction and stroke prevalence in female participants.

\begin{tabular}{|c|c|c|c|}
\hline Characteristics & Self-reported & Cotinine-verified & Survey-cotinine verified \\
\hline \multicolumn{4}{|l|}{ Age } \\
\hline$<50$ & Reference & Reference & Reference \\
\hline $50-59$ & $5.46(2.32-12.87)$ & $4.98(2.11-11.77)$ & 5.35 (2.27-12.57) \\
\hline $60-69$ & $8.80(3.58-21.64)$ & 7.87 (3.18-19.49) & 8.56 (3.49-20.99) \\
\hline$\geq 70$ & $13.25(5.28-33.30)$ & $11.91(4.71-30.12)$ & $12.83(5.13-32.11)$ \\
\hline \multicolumn{4}{|l|}{ Marital status } \\
\hline Married & Reference & Reference & Reference \\
\hline Single (separated or divorced) & $0.86(0.60-1.23)$ & $0.91(0.65-1.29)$ & $0.87(0.61-1.24)$ \\
\hline Never-married & $0.74(0.17-3.25)$ & $0.75(0.16-3.45)$ & $0.74(0.17-3.20)$ \\
\hline \multicolumn{4}{|l|}{ Employment status } \\
\hline Employed & Reference & Reference & Reference \\
\hline Unemployed & $2.00(1.46-2.74)$ & $2.04(1.49-2.80)$ & $2.01(1.47-2.76)$ \\
\hline \multicolumn{4}{|l|}{ Education } \\
\hline$<$ High school & $2.06(0.99-4.29)$ & $2.09(1.01-4.35)$ & $2.08(1.01-4.33)$ \\
\hline High school & $143(0.71-2.88)$ & $1.44(0.72-2.90)$ & $1.45(0.72-2.92)$ \\
\hline$>$ High school & Reference & Reference & Reference \\
\hline \multicolumn{4}{|l|}{ Monthly family income } \\
\hline$<25^{\text {th }}$ & $1.79(1.09-2.91)$ & $1.84(1.13-3.00)$ & $1.80(1.10-2.94)$ \\
\hline $25-50^{\text {th }}$ & $1.62(0.98-2.67)$ & $1.61(0.98-2.66)$ & $1.63(0.99-2.68)$ \\
\hline $50-75^{\text {th }}$ & $1.59(0.95-2.67)$ & $1.61(0.96-2.69)$ & $1.60(0.95-2.67)$ \\
\hline$\geq 75^{\text {th }}$ & Reference & Reference & Reference \\
\hline \multicolumn{4}{|l|}{ Number of household members } \\
\hline 1 & $1.12(0.63-1.98)$ & $1.11(0.63-1.94)$ & $1.12(0.63-1.98)$ \\
\hline 2 & $1.06(0.61-1.85)$ & $1.06(0.61-1.84)$ & $1.07(0.61-1.86)$ \\
\hline 3 & $0.91(0.51-1.64)$ & $0.90(0.50-1.61)$ & $0.92(0.51-1.65)$ \\
\hline 4 & $0.77(0.40-1.49)$ & $0.76(0.40-1.46)$ & $0.78(0.40-1.49)$ \\
\hline$\geq 5$ & Reference & Reference & Reference \\
\hline \multicolumn{4}{|l|}{ Residence area } \\
\hline Urban & Reference & Reference & Reference \\
\hline Rural & $1.21(0.90-1.63)$ & $1.19(0.88-1.59)$ & $1.21(0.90-1.63)$ \\
\hline \multicolumn{4}{|l|}{ Smoking status } \\
\hline \multicolumn{4}{|l|}{ Self-reported } \\
\hline Non-smoker & Reference & - & - \\
\hline Ex-smoker & $2.74(1.59-4.71)$ & - & - \\
\hline Smoker & $0.96(0.53-1.72)$ & - & - \\
\hline \multicolumn{4}{|l|}{ Cotinine-verified } \\
\hline Non-smoker & - & Reference & - \\
\hline Smoker & - & $1.19(0.88-1.59)$ & - \\
\hline \multicolumn{4}{|l|}{ Survey-cotinine verified } \\
\hline Non-smoker & - & - & Reference \\
\hline Ex-smoker & - & - & $2.92(1.64-5.18)$ \\
\hline Smoker & - & - & $0.90(0.56-1.44)$ \\
\hline \multicolumn{4}{|l|}{ Body mass index } \\
\hline$<23$ & Reference & Reference & Reference \\
\hline $23-25$ & $1.09(0.77-1.56)$ & $1.08(0.76-1.54)$ & $1.08(0.76-1.54)$ \\
\hline$\geq 25$ & $1.05(0.77-1.44)$ & $1.06(0.78-1.44)$ & $1.05(0.77-1.43)$ \\
\hline \multicolumn{4}{|l|}{ Hypertension } \\
\hline No & Reference & Reference & Reference \\
\hline Yes & $2.43(1.80-3.28)$ & $2.41(1.78-3.25)$ & $2.43(1.80-3.28)$ \\
\hline \multicolumn{4}{|l|}{ Diabetes } \\
\hline No & Reference & Reference & Reference \\
\hline Yes & $1.29(0.97-1.74)$ & $1.31(0.98-1.75)$ & $1.29(0.96-1.73)$ \\
\hline
\end{tabular}

Adjusted for age, marital status, employment status, education level, monthly family income, number of household members, residence area, smoking status, body mass index, hypertension and diabetes. 
Cigarette smoke contains over 4000 compounds, many of which are extremely reactive and affect the physiology of several systems in the body. These compounds include nicotine, tar, carbon monoxide, and nitrogen oxide [14].

Nicotine can elevate BP via various biological mechanisms: sympathomimetic action, modulation of the renin-angiotensin system, and acute vasopressor effects. All of these mechanisms are associated with increases in inflammatory markers through the upregulation of arginine vasopressin and endothelin-1 [15]. Nicotine can also increase low-density lipoprotein and decrease high-density lipoprotein, thereby accelerating the progression of atherosclerosis [2].

Carbon monoxide and hemoglobin combine to produce carboxy-hemoglobin, which induces hypoxia, increases in the number of red blood cells, and increases in blood viscosity, thereby, inducing thrombosis and atherosclerosis [16]. Through these mechanisms, structural damage to the arterial walls from smoking is believed to cause MI and induce stroke.

Previous studies have shown that current smoking status is significantly associated with MI [5, 6] and stroke [7, 17], but the present study has not shown a significant association. Proposed herein, is that the differing results may be due to differences in study design. The study design of the previous studies were case-control and population-based prospective cohorts; the present study design was cross-sectional. Other Korean and Polish studies that used cross-sectional designs showed similar results to the current study [8, 9, 18]. A Korean study using the 2009 Community Health Survey data gathered by the Korea Centers for Disease Control and Prevention showed current smoking was not associated with physician-diagnosed MI (OR: 0.96; 95\% CI: 0.82-1.12) and stroke (OR: 0.84; 95\% CI: 0.74-0.94), but ex-smoking was associated with physician-diagnosed MI (OR: 1.45; 95\% CI: 1.26-1.67) and stroke (OR: 1.38; 95\% CI: 1.24-1.53) [9]. In the Polish study, the researchers showed a significant association between former smokers and CVD (OR: 1.33; 95\% CI: 1.05-1.68), but not between current smokers and CVD (OR: 1.06; 95\% CI: 0.77-1.47) [18]. In addition, after cardiovascular events, patients were advised to quit smoking by physicians [19]. Therefore, the idea that cross-sectional studies, including the present study, might show no association between current smoking and CVD, but a significant association between ex-smoking and CVD.
For stroke, previous Korean studies showed different results. One study showed that ex-smoking status was associated with stroke in Korean male adults, but current smoking was not [9]. Another study showed that current smoking status was associated with stroke (OR: 1.060; 95\% CI: 1.022-1.100) [8]. Because the prevalence of stroke increases with age, it was assumed these differing results may be due to differences in participant age. One study included male subjects 30 years or older [9], while another study included subjects 50 years or older [8]. In the current study, participants 19 years or older were included and showed that ex-smoking status, but not current smoking status, was associated with stroke.

In the present study, female ex-smoking status had higher odds ratio for CVD than males, compared to non-smoking status. Two hypotheses are herein proposed. First, the use of oral contraceptives and postmenopausal hormone replacement therapy in female smokers might increase CVD incidence, and the association between ex-smoker and CVD might be higher in female smokers due to smoking cessation after a CVD attack. The use of oral contraceptives and postmenopausal hormonal replacement therapy in smokers was known to increase the risk of MI and stroke [20-22]. Although the use of oral contraceptives did not increase the risk of MI in non-smokers, the use of oral contraceptives significantly increased the risk of $\mathrm{MI}$ in smokers [23]. The use of oral contraceptives also showed a higher prevalence of stroke in smokers compared to non-smokers [20], and the use of postmenopausal hormonal replacement therapy showed the same results for MI and stroke [21, 22]. Second, it was assumed these effects might be due to more female hidden smokers than males. In the case of self-reported ex-smokers, the probability of being identified as cotinine-verified smokers was $10.46 \%$ for men and $18.6 \%$ for women.

In the present study, the ratios of cotinineverified to self-reported smoking rates were 1.95 for women and 1.08 for men. These rates were similar to the findings of a previous Korean study [10]. That study reported that ratios of cotinine-verified to self-reported smoking rates were 2.36 for women and 1.12 for men [10], but studies in other countries reported no sex differences in underreporting the rate of smoking history [24, 25].

In a study from the United States, the rates of agreement between self-reported and cotinine-verified smokers were $91.6 \%$ for women and $89.7 \%$ for men [24]. Additionally, a study from 
Finland documented that $2.5 \%$ of men and $2.7 \%$ of women who self-reported as non-smokers had positive serum cotinine levels [25].

This result means there were more hidden female smokers than hidden male smokers in the current study. It was assumed that self-reported smoking in Korean women underestimates the true prevalence as a result of Confucianism. The adoption of Confucianism can result in a patriarchal culture in which female smoking is stigmatized [26]. The discrepancy in the underreporting rates between the sexes could lead to statistical inconsistencies.

To better understand the association between CVD and smoking, a new variable was created; SCS, in order to consider the effects of hidden smoking and other types of smoking. This new variable showed similar results compared to self-reported smoking status. Therefore, it isplausable to suggest that passive and light smoking may affect CVD development and are similar to affects of active smoking.

Light and social smokers often are not detected; many of these individuals have the perception of being non-smokers [27, 28]. However, a recent study reported that social smokers had significantly higher risks of CVD than non-smokers. Moreover, no significant differences in the development of hypertension have been reported between social smokers and current smokers [29]. Another report showed that light smoking was associated with a significantly higher risk of dying from ischemic heart disease [30]. With respect to the relationship between CVD and social and light smoking, a stable pattern of chronic low-level consumption may be assumed to have similar effects on CVD as constant, current active smoking.

\section{Limitations of the study}

There are several limitations to this study. First, because this study was based on a survey, there may be selection and recall biases. Second, because this study was cross-sectional in design, a causal relationship between smoking and CVD could not be confirmed. Third, although CVD was defined as MI and stroke in this study, CVD also includes other coronary heart diseases such as angina and peripheral arterial diseases; this was considered to be a limitation in the present study. Finally, potential confounding factors, including amount and duration of smoking, diet patterns, and genetic or sex variations affecting nicotine metabolism, still exist. Further prospective and collaborative worldwide studies are needed to clarify the effect of hidden female smoking on CVD.
However, the strength of this study is its use of nationally and widely sampled data to assess sexspecific relationships between smoking status and CVD through the creation of a new variable, SCS. This new variable was used to evaluate the effect of hidden smoking on CVD.

\section{Statement of ethics}

This study was approved by the Institutional Review Board (IRB) of Samsung Changwon Hospital (IRB No: SCMC2019-04-005). Informed consent was waived by the board.

\section{Conclusions}

This large observational study found that ex-smoking status was associated with CVD and female ex-smoking status had a higher adjusted odds ratio for CVD than males compared to nonsmoking status. In addition, there was an effect of hidden female smoking on the association between smoking status and CVD in Korean adults.

\section{Conflict of interest: None declared}

\section{References}

1. McEvoy JW, Blaha MJ, DeFilippis AP, et al. Cigarette smoking and cardiovascular events: role of inflammation and subclinical atherosclerosis from the MultiEthnic Study of Atherosclerosis. Arterioscler Thromb Vasc Biol. 2015; 35(3): 700-709, doi: 10.1161/ ATVBAHA.114.304562, indexed in Pubmed: 25573855.

2. Bullen C. Impact of tobacco smoking and smoking cessation on cardiovascular risk and disease. Expert Rev Cardiovasc Ther. 2008; 6(6): 883-895, doi: 10.1586/14779072.6.6.883, indexed in Pubmed: 18570625.

3. Mons U, Müezzinler A, Gellert C, et al. Impact of smoking and smoking cessation on cardiovascular events and mortality among older adults: meta-analysis of individual participant data from prospective cohort studies of the CHANCES consortium. BMJ. 2015; 350: h1551, doi: 10.1136/bmj.h1551, indexed in Pubmed: 25896935.

4. Aune D, Schlesinger S, Norat T, et al. Tobacco smoking and the risk of sudden cardiac death: a systematic review and meta-analysis of prospective studies. Eur J Epidemiol. 2018; 33(6): 509-521, doi: 10.1007/s10654-017-0351-y, indexed in Pubmed: 29417317.

5. Yusuf S, Hawken S, Ounpuu S, et al. Effect of potentially modifiable risk factors associated with myocardial infarction in 52 countries (the INTERHEART study): case-control study. Lancet. 2004; 364(9438): 937-952, doi: 10.1016/S0140-6736(04)17018-9, indexed in Pubmed: 15364185.

6. Millett ERC, Peters SAE, Woodward M. Sex differences in risk factors for myocardial infarction: cohort study of UK Biobank participants. BMJ. 2018; 363: k4247, doi: 10.1136/bmj.k4247, indexed in Pubmed: 30404896.

7. Peters SAE, Huxley RR, Woodward M. Smoking as a risk factor for stroke in women compared with men: a systematic review and meta-analysis of 81 cohorts, including 3,980,359 individuals and 
42,401 strokes. Stroke. 2013; 44(10): 2821-2828, doi: 10.1161/ STROKEAHA.113.002342, indexed in Pubmed: 23970792.

8. Lee W, Hwang SH, Choi H, et al. The association between smoking or passive smoking and cardiovascular diseases using a Bayesian hierarchical model: based on the 2008-2013 Korea Community Health Survey. Epidemiol Health. 2017; 39: e2017026, doi: 10.4178/epih.e2017026, indexed in Pubmed: 28728350.

9. Chang S, Kim H, Kim V, et al. Association between smoking and physician-diagnosed stroke and myocardial infarction in male adults in Korea. Int J Environ Res Public Health. 2016; 13(2): 158, doi: 10.3390/ijerph13020158, indexed in Pubmed: 26821036.

10. Jung-Choi KH, Khang YH, Cho HJ. Hidden female smokers in Asia: a comparison of self-reported with cotinine-verified smoking prevalence rates in representative national data from an Asian population. Tob Control. 2012; 21(6): 536-542, doi: 10.1136/tobaccocontrol-2011-050012, indexed in Pubmed: 21972062.

11. Kim SH, Lee JS. The association of smoking and hypertension according to cotinine-verified smoking status in 25,150 Korean adults. Clin Exp Hypertens. 2019; 41(5): 401-408, doi: 10.1080/1 0641963.2018.1489548, indexed in Pubmed: 30059635.

12. noue S, Zimmet P, Caterson I, et al. The Asia-Pacific perspective: redefining obesity and its treatment. 2000. http://iotf. org (accessed 19 February 2002).

13. Haufroid V, Lison D. Urinary cotinine as a tobacco-smoke exposure index: a minireview. Int Arch Occup Environ Health. 1998; 71(3): 162-168, doi: 10.1007/s004200050266, indexed in Pubmed: 9591157.

14. Guerin MR, Higgins CE, Griest WH. The analysis of the particulate and vapour phases of tobacco smoke. IARC Sci Publ. 1987(81): 115-139, indexed in Pubmed: 3323048.

15. Grassi G, Seravalle G, Calhoun DA, et al. Mechanisms responsible for sympathetic activation by cigarette smoking in humans. Circulation. 1994; 90(1): 248-253, doi: 10.1161/01.cir.90.1.248, indexed in Pubmed: 8026005.

16. Ambrose JA, Barua RS. The pathophysiology of cigarette smoking and cardiovascular disease: an update. J Am Coll Cardiol. 2004; 43(10): 1731-1737, doi: 10.1016/j.jacc.2003.12.047, indexed in Pubmed: 15145091.

17. Xu L, Schooling CM, Chan WM, et al. Smoking and hemorrhagic stroke mortality in a prospective cohort study of older Chinese. Stroke. 2013; 44(8): 2144-2149, doi: 10.1161/STROKEAHA.113.001500, indexed in Pubmed: 23723306.

18. Islami F, Mańczuk M, Vedanthan R, et al. A cross-sectional study of cardiovascular disease and associated factors. Ann Agric Environ Med. 2011; 18(2): 255-259, indexed in Pubmed: 22216792.

19. Prochaska JJ, Benowitz NL. Smoking cessation and the cardiovascular patient. Curr Opin Cardiol. 2015; 30(5): 506-511, doi: 10.1097/HCO.0000000000000204, indexed in Pubmed: 26196657.
20. Farley TM, Meirik O, Chang CL, et al. Combined oral contraceptives, smoking, and cardiovascular risk. J Epidemiol Community Health. 1998; 52(12): 775-785, doi: 10.1136/jech.52.12.775, indexed in Pubmed: 10396518.

21. Chilvers CED, Knibb RC, Armstrong SJ, et al. Post menopausal hormone replacement therapy and risk of acute myocardial infarction--a case control study of women in the East Midlands, UK. Eur Heart J. 2003; 24(24): 2197-2205, doi: 10.1016/j. ehj.2003.09.019, indexed in Pubmed: 14659771.

22. Li C, Engström G, Hedblad Bo, et al. Risk of stroke and hormone replacement therapy. A prospective cohort study. Maturitas. 2006; 54(1): 11-18, doi: 10.1016/j.maturitas.2005.10.002, indexed in Pubmed: 16321486.

23. Croft P, Hannaford PC. Risk factors for acute myocardial infarction in women: evidence from the Royal College of General Practitioners' oral contraception study. BMJ. 1989; 298(6667): 165-168, doi: 10.1136/bmj.298.6667.165, indexed in Pubmed: 2493841 .

24. Assaf AR, Parker D, Lapane KL, et al. Are there gender differences in self-reported smoking practices? Correlation with thiocyanate and cotinine levels in smokers and nonsmokers from the Pawtucket Heart Health Program. J Womens Health (Larchmt). 2002; 11(10): 899-906, doi: 10.1089/154099902762203731, indexed in Pubmed: 12630407.

25. Vartiainen E, Seppälä T, Lillsunde $P$, et al. Validation of self reported smoking by serum cotinine measurement in a community-based study. J Epidemiol Community Health. 2002; 56(3): 167-170, doi: 10.1136/jech.56.3.167, indexed in Pubmed: 11854334

26. Park M, Chesla C. Revisiting Confucianism as a conceptual framework for Asian family study. J Fam Nurs. 2007; 13(3): 293-311, doi: 10.1177/1074840707304400, indexed in Pubmed: 17641110

27. Schane RE, Glantz SA, Ling PM. Social smoking implications for public health, clinical practice, and intervention research. Am J Prev Med. 2009; 37(2): 124-131, doi: 10.1016/j.amepre.2009.03.020, indexed in Pubmed: 19589449.

28. Husten CG. How should we define light or intermittent smoking? Does it matter? Nicotine Tob Res. 2009; 11(2): 111-121, doi: 10.1093/ntr/ntp010, indexed in Pubmed: 19246425.

29. Gawlik KS, Melnyk BM, Tan A. An epidemiological study of population health reveals social smoking as a major cardiovascular risk factor. Am J Health Promot. 2018; 32(5): 1221-1227, doi: 10.1177/0890117117706420, indexed in Pubmed: 28464696.

30. Bjartveit K, Tverdal A. Health consequences of smoking 1-4 cigarettes per day. Tob Control. 2005; 14(5): 315-320, doi: 10.1136/ tc.2005.011932, indexed in Pubmed: 16183982. 